\title{
Charge-induced optical bistability in thermal Rydberg vapor
}

\author{
Daniel Weller, Alban Urvoy, Andy Rico, Robert Löw, and Harald Kübler* \\ 5. Physikalisches Institut and Center for Integrated Quantum Science and Technology, University of Stuttgart, Pfaffenwaldring 57, \\ 70569 Stuttgart, Germany
}

(Received 20 September 2016; published 8 December 2016)

\begin{abstract}
We investigate the phenomenon of optical bistability in a driven ensemble of Rydberg atoms. By performing two experiments with thermal vapors of rubidium and cesium, we are able to shed light on the underlying interaction mechanisms causing such a nonlinear behavior. Due to the different properties of these two atomic species, we conclude that the large polarizability of Rydberg states in combination with electric fields of spontaneously ionized Rydberg atoms is the relevant interaction mechanism. In the case of rubidium, we directly measure the electric field in a bistable situation via two-species spectroscopy. In cesium, we make use of the different sign of the polarizability for different $l$ states and the possibility of applying electric fields. Both these experiments allow us to rule out dipole-dipole interactions and support our hypothesis of a charge-induced bistability.
\end{abstract}

DOI: 10.1103/PhysRevA.94.063820

\section{INTRODUCTION}

The extraordinary properties of highly excited atoms have led to a renaissance in the research field of Rydberg atoms. Especially the strong interaction between Rydberg atoms in combination with ultracold gases paved the way for a variety of applications and novel phenomena, among which are quantum gates [1,2], quantum phase transitions [3,4], optical nonlinearities on the single-photon level [5-9], beyond twobody interactions [10], excitation transfer [11,12], aggregation of excitations [13-15], and ultra-long-range molecules [16]. Although most of the recent experimental results originate from ultracold atoms, it is sometimes advantageous to study Rydberg atoms in thermal vapor, especially when large atom numbers or high number densities are required-as is the case for electric field sensing [17], aggregation [15], or optical bistabilities (detailed description in Sec. III). Specifically the latter has exclusively been studied in thermal vapors [18-22]. In these publications, one interpretation suggests that the underlying mechanism for the bistability is a dipole-dipole interaction between Rydberg states. Our experimental findings presented here however support an alternative interpretation: We argue that the large polarizability of Rydberg states in the presence of ions is at the heart of the optical bistability in thermal Rydberg vapor.

In this paper, we describe the observation of optical bistability in two separate experiments, one with a thermal vapor of rubidium atoms and the other with cesium. We show that the underlying interaction shift is caused by charges in the medium, themselves being produced by spontaneous ionization of Rydberg atoms. In the former case, we make use of the natural abundance of the two distinct rubidium isotopes. While ${ }^{85} \mathrm{Rb}$ atoms are driven to bistability, a simultaneous spectroscopic measurement on the coexisting ${ }^{87} \mathrm{Rb}$ isotope

\footnotetext{
*h.kuebler@physik.uni-stuttgart.de

Published by the American Physical Society under the terms of the Creative Commons Attribution 3.0 License. Further distribution of this work must maintain attribution to the author(s) and the published article's title, journal citation, and DOI.
}

shows a large broadening and shift of the Rydberg resonance. This observation is consistent with the presence of ions in the medium, induced by the excitation of Rydberg atoms. In the case of ${ }^{133} \mathrm{Cs}$, we observe a strong sensitivity of the optical bistability features to external ac electric fields, strongly indicating that charged particles in the medium are closely related to the phenomenon. By compiling the signs of the interaction shifts for various species and Rydberg states, we finally conclude that the observed optical bistability is only compatible with interactions between Rydberg atoms and charges, and a dipole-dipole interaction between Rydberg atoms is ruled out as a possible scenario.

The paper is organized as follows. In Sec. II we describe the two experimental setups and the implementation of the measurements performed. Because we used two different elements, the experiment with two rubidium isotopes is detailed first, before we introduce the setup involving cesium. Section III then contains our analysis and argumentation in the same order, followed by the conclusion in Sec. IV.

\section{MATERIALS AND METHODS}

\section{A. Rubidium}

We drive an optical bistability in ${ }^{85} \mathrm{Rb}$ similar to the work in Ref. [18], using an EIT-like excitation scheme [23] — depicted in Fig. 1(a) and referred to as $\mathrm{EIT}_{\mathrm{OB}}$. A frequency-stabilized 795-nm laser probes the ${ }^{85} \mathrm{Rb} 5 S_{1 / 2}, F=3 \rightarrow 5 P_{1 / 2}, F^{\prime}=2$ transition on resonance with a Rabi frequency of $\Omega_{795}=$ $2 \pi \times 37 \mathrm{MHz}$, while a 477-nm laser is scanned over the transition $5 P_{1 / 2} \rightarrow 32 S_{1 / 2}$. For the $477-\mathrm{nm}$ laser, the maximal Rabi frequency is $2 \pi \times 25 \mathrm{MHz}$, limited by the available laser power. The two laser beams are focused to a waist of $40 \mu \mathrm{m}$ and overlapped in a copropagating configuration. This results in a simple Lorentzian-like excitation spectrum, in contrast to the counterpropagating configuration [23-25]. Due to population shelving [26], the transmission signal of the 795-nm laser is roughly proportional to the population of the Rydberg state. The transmission of the 795-nm laser is monitored as a function of the detuning $\Delta_{477}$, recording both directions of the frequency scan (from red to blue, and vice versa). 


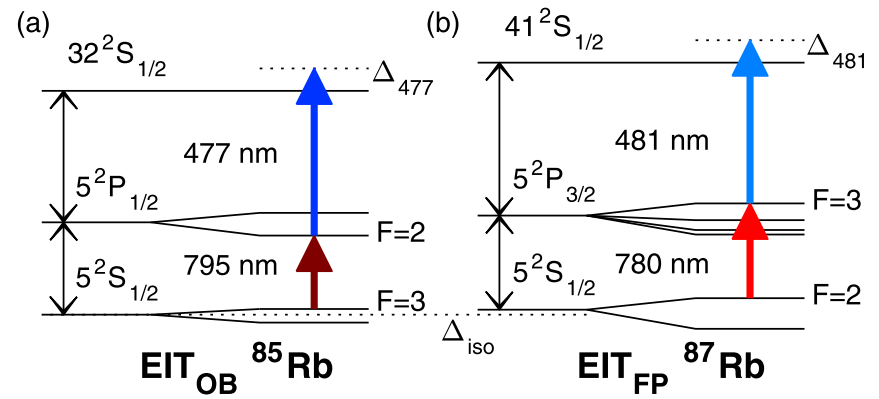

FIG. 1. Relevant transitions in the resonant two-photon excitation schemes for both isotopes. (a) EIT $_{\mathrm{OB}}$ drives the optical bistability. (b) EIT $_{\mathrm{FP}}$ acts as a probe for electric fields.

Figure 2 shows the experimental setup. A 2-mm-thick glass cell containing the rubidium vapor is placed at the focus of the beams. The cell is a spectroscopy cuvette (quartz glass, Hellma Analytics) connected to a reservoir and filled with a droplet of naturally occurring rubidium (i.e., $78 \%{ }^{85} \mathrm{Rb}$ and $22 \%{ }^{87} \mathrm{Rb}$ ) under vacuum. The temperature of the reservoir is stabilized to control the vapor density, while the higher cell temperature $\left(T_{\text {res. }}=80^{\circ} \mathrm{C}\right.$ to $\left.120^{\circ} \mathrm{C}, T_{\text {cell }}=135^{\circ} \mathrm{C}\right)$ prevents unwanted condensation of the alkali. By measuring the absorption in the $D_{1}$ line, the vapor density is determined as $\mathcal{N}_{85}=1.8 \times 10^{12} \mathrm{~cm}^{-3}$ and $\mathcal{N}_{87}=0.7 \times 10^{12} \mathrm{~cm}^{-3}$ for ${ }^{85} \mathrm{Rb}$ and ${ }^{87} \mathrm{Rb}$, respectively.

In order to probe into the mechanisms and the cause of the optical bistability, we simultaneously measure an additional EIT-like spectrum [23] on the less abundant isotope, ${ }^{87} \mathrm{Rb}$, hereafter named $\mathrm{EIT}_{\mathrm{FP}}$ (field probe). The advantage of using two different isotopes is that-except for interspecies interactions - the two schemes are completely decoupled due to the different wavelengths of the transitions. Similar to the excitation scheme EIT $_{\mathrm{OB}}$ presented above, we use two lasers at $780 \mathrm{~nm}$ (probe, $\Omega_{780}=2 \pi \times 66 \mathrm{MHz}$ ) and $481 \mathrm{~nm}$ (coupling, $\Omega_{481}=2 \pi \times 10 \mathrm{MHz}$ ) to drive the ladder scheme $5 S_{1 / 2}, F=2 \rightarrow 5 P_{3 / 2}, F^{\prime}=3 \rightarrow 41 S_{1 / 2}$ of ${ }^{87} \mathrm{Rb}$ [see Fig. 1(b)]. Again, the 780-nm laser is locked on resonance and the $481-\mathrm{nm}$ laser has a variable detuning $\Delta_{481}$ with respect to the upper transition. These two additional laser beams are overlapped with the previous pair and in the same arrangement as for $\mathrm{EIT}_{\mathrm{OB}}$ up to the following: the 780- and 481-nm lasers are counterpropagating and the polarizations are perpendicular to those of $\mathrm{EIT}_{\mathrm{OB}}$. By independently scanning $\Delta_{481}$ and $\Delta_{477}$, we obtain two-dimensional transmission spectra of the 780-nm probe laser, an example of which is shown in Fig. 6(a). Since the choice of Rabi frequencies compromises between a narrow linewidth and signal visibility, the signal-to-noise ratio is improved by amplitude-modulating the 481-nm laser with a frequency of $30 \mathrm{kHz}$ and demodulating the transmission signal using a lock-in amplifier (Femto, LIA-MV-200).

\section{B. Cesium}

In the experiment based on ${ }^{133} \mathrm{Cs}$, we address the atoms with the two-photon ladder scheme $6 S_{1 / 2}, F=3 \rightarrow 7 P_{1 / 2}, F^{\prime}=$ $4 \rightarrow n l$ as depicted in Fig. 3(a). The Rabi frequencies for the lower transition at $459 \mathrm{~nm}$ and the upper transition at 1070 $\mathrm{nm}$ are set to $\Omega_{459}=2 \pi \times 6 \mathrm{MHz}$ and $\Omega_{1070}=2 \pi \times 146$ $\mathrm{MHz}$, respectively. Similar to the rubidium experiment, the transmission of the 459-nm laser is measured directly on a photodiode while the 1070 -nm detuning $\Delta_{1070}$ is varied. The measurements are performed using counterpropagating lasers, focused to $50 \mu \mathrm{m}$ in a $3-\mathrm{mm}$ vapor cell $\left(\mathcal{N}_{\mathrm{Cs}}=1.2 \times 10^{13}\right.$ $\mathrm{cm}^{-3}$ ), as sketched in Fig. 3(b). In this inverted level scheme, where the wavelength of the lower transition is smaller than the one for the upper transition, additional decay channels and transit time broadening result in enhanced absorption [25]. With the use of a fiber amplifier, we can reach higher Rabi frequencies on the upper transition with cesium than in our rubidium setup. Furthermore, we can select various principal and two different azimuthal quantum numbers $(n=$ 20 to $60, l=S, D)$ for the Rydberg state, thus examining the response of systems with different properties. In the following, we consider in particular the Rydberg states $23 D_{3 / 2}$ and $28 S_{1 / 2}$.

The major additional feature available in this setup is the ability to externally apply an electric field across the cell. The glass cell is placed between two electrodes that produce an electric field roughly parallel to the polarization of the laser beams. A specially designed strip-line guides the applied microwave (possible frequencies range from $\mathrm{dc}$ to several $\mathrm{GHz}$ ) to an electrode next to the spectroscopy cell. The electrode on the other side is terminated with $50 \Omega$ [Fig. 3(b)]. For our measurements, we inject a sine wave with frequencies of 10 to $500 \mathrm{MHz}$, yielding an oscillating electric field between the electrodes with an amplitude of approximately $3.2 \mathrm{~V} \mathrm{~cm}^{-1}$.

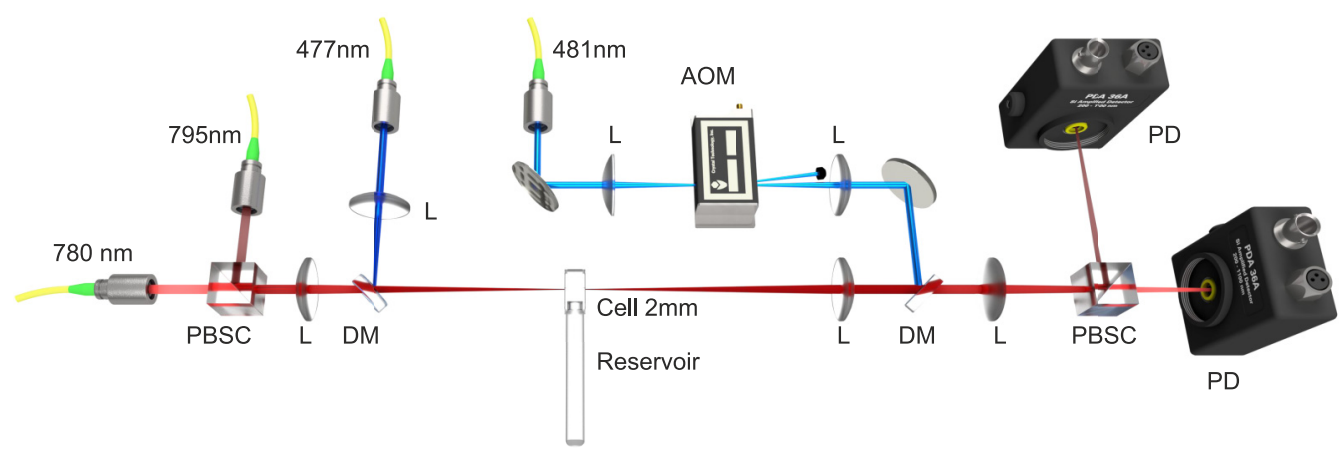

FIG. 2. Setup of the rubidium experiment. Two pairs of lasers drive the two isotopes in the same volume using an EIT-like excitation scheme (see Fig. 1). One drives an optical bistability with the scheme EIT $_{\mathrm{OB}}$, while the other probes for local fields with the scheme EIT $_{\mathrm{FP}}$. 
(a)

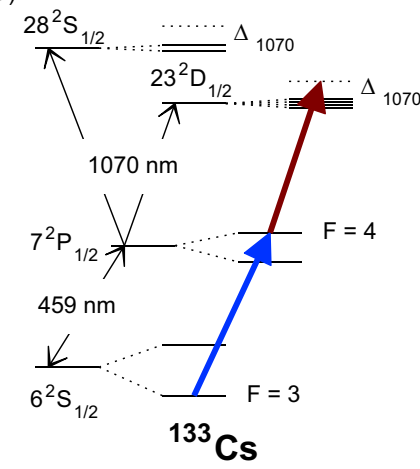

(b)

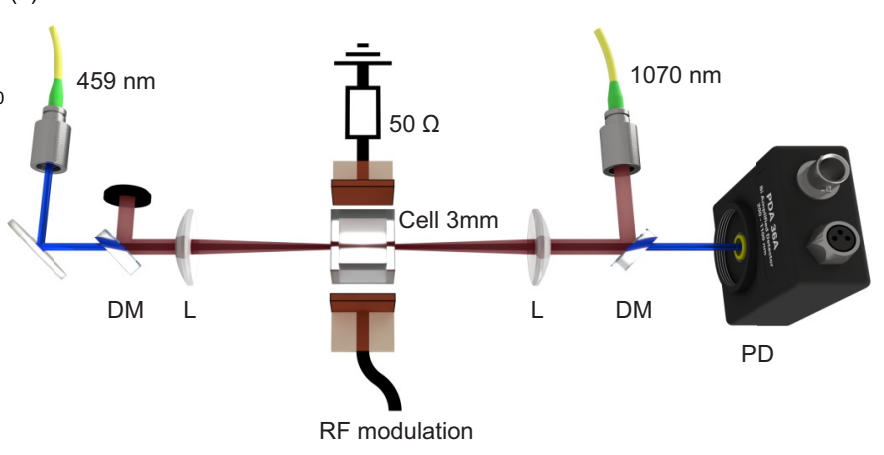

FIG. 3. Setup of the cesium experiment. (a) Level scheme with the relevant cesium transitions. (b) Counterpropagating spectroscopy setup with a 3-mm-thick cesium vapor sample. The cell is placed between two electrodes, where the modulation is applied on one port while the other is $50 \Omega$ terminated. The laser polarization is parallel to the electric field between the plates.

\section{RESULTS AND DISCUSSION}

\section{A. Rubidium}

Figure 4(a) shows the typical traces of the EIT ${ }_{\mathrm{OB}}$ system. For increasing Rabi frequencies of the coupling laser, the EIT $_{\mathrm{OB}}$ peak is shifted to the red and becomes more and more distorted, until at sufficiently large intensity the system becomes bistable, as in Ref. [18]. This phenomenon is the result of a competition between a nonlinear energy shift due to an interaction effect, which is dependent on the Rydberg state population, and decay from the Rydberg state. On the one hand, when the frequency is scanned from the blue detuned side to the red, there is a buildup of Rydberg population that sustains the ability to excite Rydberg atoms even away from
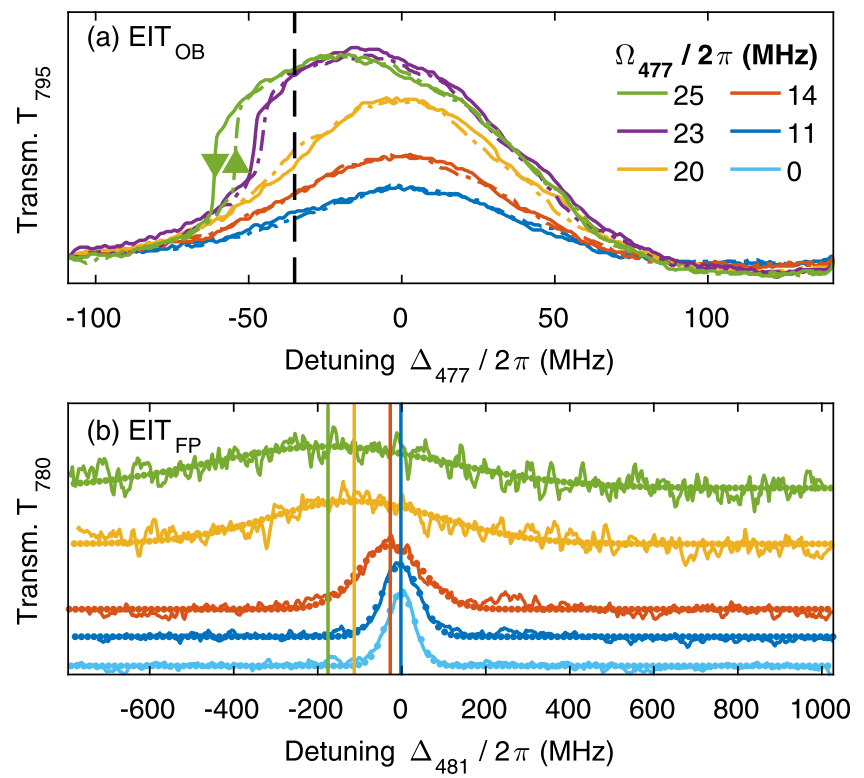

FIG. 4. Rubidium measurements. (a) Deformation of the 795-nm laser $\left(\mathrm{EIT}_{\mathrm{OB}}\right)$ transmission peak towards optical bistability. Scans across the resonance from blue to red detuning (dashed) and reverse (solid line). (b) $\mathrm{EIT}_{\mathrm{FP}}$ scans taken at a fixed detuning $\Delta_{477} / 2 \pi=$ $-35 \mathrm{MHz}$ [vertical dashed line in panel (a)]. The feature is shown for different Rabi frequencies of the EIT $_{\mathrm{OB}}$ system (offset to discern the lines). resonance. When the detuning becomes too large, the decay mechanisms prevail and the population breaks down. This results in a sudden change of the transmission level, as can be observed in the solid lines in Fig. 4(a). On the other hand, when the frequency is scanned to the blue (dashed lines), the Rydberg population stays low in the bistable region until the detuning becomes small enough to sufficiently excite atoms. These atoms act like a seed for subsequent excitations. When a certain threshold is reached, a sudden increase in population is triggered and the system is switched to the high population state. Overall, a hysteresis in the transmission spectrum can be observed.

We make a complementary observation by probing the interatomic interactions in the excitation volume using the additional Rydberg EIT scheme EIT $_{\mathrm{FP}}$. For a fixed detuning $\Delta_{477}$, we observe the signal in the EIT $_{\mathrm{FP}}$ scheme as shown in Fig. 4(b). The traces here show a significantly growing shift for increasing EIT $_{\mathrm{OB}}$ Rabi frequencies and therefore a rising Rydberg population in the bistable scheme EIT $_{\mathrm{OB}}$. In Figs. 4(a) and 4(b), besides a broadening of the lines, the sign of the shifts in both schemes clearly indicates attractive interactions.

\section{Rydberg-Rydberg interactions}

Let us assume that Rydberg-Rydberg interactions (e.g., dipole-dipole or van-der-Waals) are the underlying mechanism for the optical bistability. Then, the interactions between $32 \mathrm{~S}$ and $32 S$ states on the one hand should explain the bistability itself, and, on the other hand, those between $41 S$ and $32 S$ are relevant for the shifts measured with EIT $_{\mathrm{FP}}$. Figures 5(a) and 5(b) show computed pair-potentials for the interactions of the $32 S$ state with the $32 S$ and $41 S$ states, respectively. The potentials are calculated up to dipole-quadrupole terms similar to those in Ref. [27]. It is noteworthy that between rubidium $S$ states, the van-der-Waals interaction potential is constantly repulsive, i.e., leading to a shift towards blue wavelengths, contrary to what we observe. In Fig. 5(c), the nearest-neighbor distance probability (given by the Chandrasekhar distribution) is plotted for various densities of Rydberg atoms. By weighting the pair-potential map with the nearest-neighbor distribution, we determine the overall sign of the expected level shift in the experiment. This gives a very rough estimate of the line shapes that one should expect from the measurements, which 


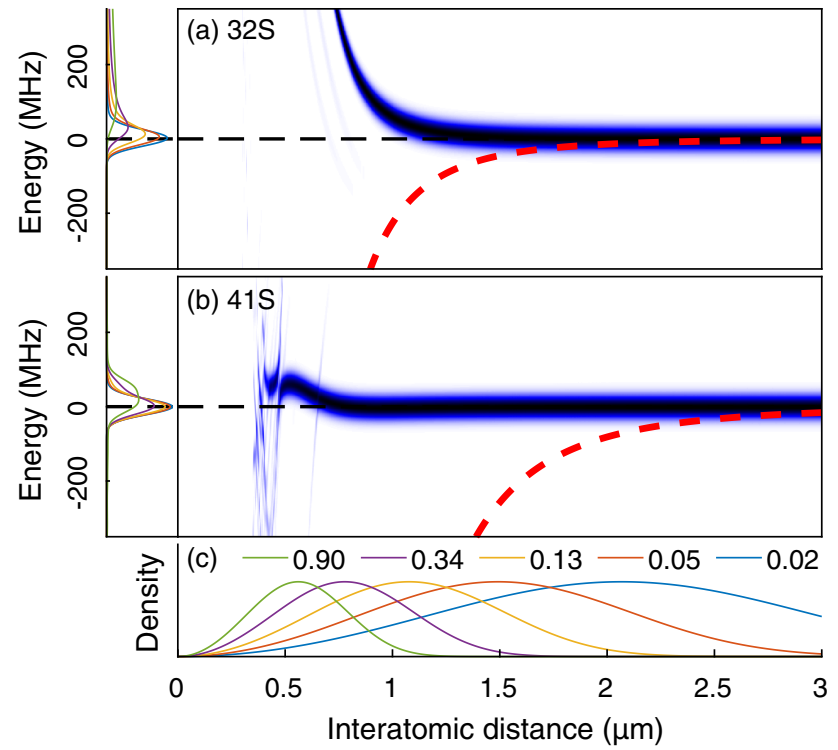

FIG. 5. Rubidium interaction potentials. (a) Pair potentials for $32 S-32 S$. The color shading represents the projection on the unperturbed pair state. (b) Pair potentials for $41 S-32 S$. The avoided crossings arise from the interaction with different pair-states (not shown here). The $C_{4} / r^{4}$ potential is plotted as a red dashed line for the $32 S$ state (a) and the $41 S$ state (b). (c) Nearest-neighbor distribution for various densities (given in $\mu \mathrm{m}^{-3}$ ) of Rydberg atoms, corresponding to Rydberg fractions of $1 \%$ to $50 \%$. The curves are normalized to the maximum density. Left: Projection of pair potentials weighted with distribution (c).

are displayed on the left in Fig. 5. Although this estimate only accounts for interactions between nearest neighbors, it is clear that only blue shifts are to be expected with this mechanism. The shift even vanishes for dilute vapors as given by our experimental parameters. An estimate for the density of Rydberg atoms is on the order of $0.05 \mu \mathrm{m}^{-3}$.

Apart from these direct interaction paths, it is possible that a significant part of the $S$-state population decays to neighboring $P$ states. Therefore, also the interactions between states $32 S$ and $31 P$, as well as between $41 S$ and $31 P$ are to be considered. In a related experiment with ultracold atoms [28], it was shown that this configuration does not lead to a shift of the excitation spectrum. Only a broadening was observed, consistent with the spatial integration of the dipole-dipole interaction potential in an isotropic medium. Also the $31 P-41 S$ dipole-dipole interaction is much weaker than the $31 P-32 S$ dipole-dipole interaction because the wave function overlap is smaller. This comes in contradiction with the experimental observation in Fig. 4 that the interaction shifts on the EIT $\mathrm{FP}_{\mathrm{FP}}$ scheme are larger than those on the EIT $_{\mathrm{OB}}$ scheme. It is therefore unlikely to explain the observed optical bistability by means of Rydberg-Rydberg interactions.

\section{Ionization-induced electric fields}

As an alternative explanation for the observed red interaction shift, we suggest that the bistability is caused by charges created by the ionization of Rydberg atoms [23,29,30]. The interaction potential between a single charge and a Rydberg atom (red dashed lines in Fig. 5) in this picture arises from
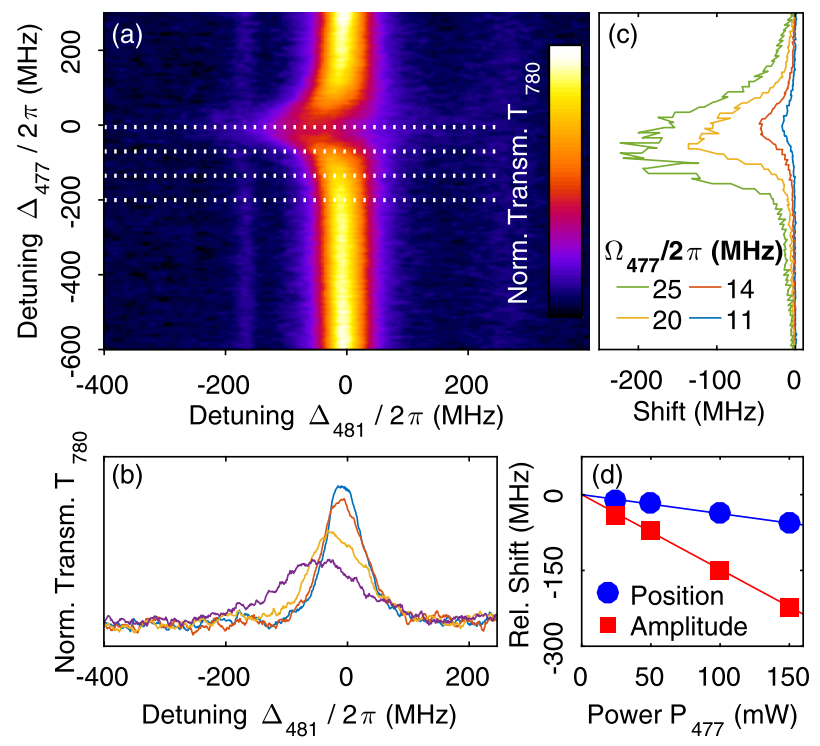

FIG. 6. Rubidium measurements. (a) Example of a twodimensional map showing the EIT $_{\mathrm{FP}}$ traces against both detunings for $\Omega_{477} / 2 \pi=14 \mathrm{MHz}$. (b) Cuts along the dashed lines in panel (a). (c) Dependence of the fitted maximum in every row in panel (a) for different Rabi frequencies $\Omega_{477}$. (d) Center frequency and amplitude of the curves in panel (c), determined via a Gaussian fit (uncertainty within marker size). The center frequency (blue dots) represents the shift in the $\mathrm{EIT}_{\mathrm{OB}}$ scheme, while the amplitude (red squares) illustrates the shift in the EIT $_{\mathrm{FP}}$ scheme. The offsets are chosen such that the linear fits intersect at zero.

the dc Stark-shift of the Rydberg state and has the form $C_{4} / r^{4}$, where $C_{4}$ is proportional to the polarizability $\alpha$ of the Rydberg state. Following the usual definition for the sign of the interaction, a positive polarizability value yields a negative energy shift. For rubidium $S$ states, $\alpha$ is invariably positive [31], thus resulting in a red shift as observed. The polarizabilities for the regarded states are computed as $\alpha_{32 S}=2.2 \mathrm{MHz} /(\mathrm{V} / \mathrm{cm})^{2}$ and $\alpha_{41 S}=12.6 \mathrm{MHz} /(\mathrm{V} / \mathrm{cm})^{2}$, respectively. Examining Fig. 4 again given these numbers, we find a good compatibility with both the sign and the magnitude of the observed shifts. Since both isotopes are exposed to the same electric field distribution in the vapor, the EIT $_{\mathrm{FP}}$ spectrum is shifted more, according to the larger polarizability $\alpha_{41 S}$.

To further substantiate our hypothesis, $\mathrm{EIT}_{\mathrm{FP}}$ traces are systematically taken for a set of detunings $\Delta_{477}$ and for various Rabi frequencies $\Omega_{477}$ in the EIT $_{\mathrm{OB}}$ scheme. The resulting data are displayed in Fig. 6. For an exemplary Rabi frequency of $\Omega_{477}=2 \pi \times 14 \mathrm{MHz}$, the resulting density plot of the transmission is shown in Fig. 6(a), along with a sample of EIT $\mathrm{FP}_{\mathrm{FP}}$ traces in Fig. 6(b). The behavior is very similar to what is shown in Fig. 4(b) for various Rabi frequencies $\Omega_{477}$, emphasizing the dependence of the EIT $_{\mathrm{FP}}$ signal on the excitation probability of $32 S$ Rydberg atoms. We can gain further insight on the relation between the two systems by evaluating the shift in the EIT $_{\mathrm{FP}}$ system relative to the unperturbed line. For a set of Rabi frequencies $\Omega_{477}$, this shift (determined by the center frequency of a Gaussian fit to the EIT $_{\mathrm{FP}}$ signal) is depicted in Fig. 6(c) against the EIT $_{\mathrm{OB}}$ detuning $\Delta_{477}$. There is a clear evolution from the unshifted 
EIT $_{\mathrm{FP}}$ signal in the off-resonant region to a maximally shifted signal, where the $\mathrm{EIT}_{\mathrm{OB}}$ system is close to resonance. Noticeably, the shift trajectories in the EIT $_{\mathrm{FP}}$ spectra show the same characteristic deformation and asymmetry towards red detuned frequencies as the transmission curves of EIT $_{\mathrm{OB}}$ in Fig. 4(a). To conclude the analysis of Fig. 6, both the amplitude and the center frequency (Gaussian fit to each curve) of the shift trajectories from panel (c) are plotted versus the Rabi frequency $\Omega_{477}$ [Fig. 6(d)]. The amplitude here directly reflects the shift in the EIT $_{\mathrm{FP}}$ system, while the position is an indirect measure for the shift in the EIT $_{\mathrm{OB}}$ system. The ratio between the slopes of the two linear fits amounts to 4:1. A qualitatively similar observation was made in Fig. 4 and again roughly reflects the ratio of polarizabilities between the states $41 S$ and $32 S$ which is $5.7: 1$.

\section{Quantitative analysis}

Let us now first estimate the ion density present in the cell and then extrapolate the necessary cross section for the ionization process, to allow a quantitative comparison to previous experiments. In the scope of ionizing collisions, the heavy particles remain quasistationary while the much lighter free electrons gain significantly more velocity. Hence, the electrons leave the volume in a much faster time scale compared to the heavier ions. In a very simplified picture neglecting the free electrons and only considering the ions, the electric field distribution in the medium is described by the so-called Holtsmark distribution [32]. The Holtsmark probability distribution function for the electric field in this case is given by

$$
\mathcal{P}(E)=H\left(E / Q_{H}\right) / Q_{H},
$$

where the normal field is given by the expression

$$
Q_{H}=\left(\frac{4}{15}\right)^{2 / 3}\left(\frac{e}{2 \epsilon_{0}}\right)\left(\mathcal{N}_{\text {ion }}\right)^{2 / 3},
$$

and

$$
H(\beta)=\frac{2}{\pi \beta} \int_{0}^{\infty} d x x \sin (x) \exp \left[-(x / \beta)^{3 / 2}\right] .
$$

An estimate of the mean ion density $\mathcal{N}_{\text {ion }}$ is found by comparing this model with the data as follows. The line shape of the measured EIT $\mathrm{FP}_{\mathrm{FP}}$ signal $S\left(\Delta_{481}\right)$ amounts to a convolution of the Holtsmark probability distribution with the EIT-signal shape $F_{\text {EIT }}\left(\Delta_{481}\right)$ :

$$
S(\Delta)=\int_{0}^{\infty} d E \mathcal{P}(E) F_{\mathrm{EIT}}\left(\Delta-\alpha E^{2} / 2\right) .
$$

In this integral, the EIT line (a $2 \pi \times 50 \mathrm{MHz}$ wide Gaussian profile [33] as in our measurements) is displaced by $-\alpha E^{2} / 2$ according to the Stark-shift. Using the approximation [34] for the Holtsmark formula, we find that the center of mass $C$ of the obtained line shape linearly scales with the ion density as

$$
C=\left(1.89 \times 10^{-9} \mathrm{cmV}^{2}\right) \alpha \mathcal{N}_{\text {ion }} .
$$

This translates to a first estimate of the observed ion density of up to $\mathcal{N}_{\text {ion }} \leqslant 1 \times 10^{10} \mathrm{~cm}^{-3}$ for an observed shift up to $2 \pi \times 250 \mathrm{MHz}$ in the EIT $_{\mathrm{FP}}$ scheme. Given the atomic ground
TABLE I. Compiled signs of Rydberg Stark-shifts, van-der-Waals interaction, and the actually observed position of the bistability.

\begin{tabular}{lccccc}
\hline \hline Element & State & vdW & Stark-shift & Bistability & Source \\
\hline${ }^{85} \mathrm{Rb}$ & $32 S_{1 / 2}$ & + & - & - & This work \\
${ }^{85} \mathrm{Rb}$ & $22 S_{1 / 2}$ & + & - & - & This work $^{\mathrm{a}}$ \\
${ }^{133} \mathrm{Cs}$ & $28 S_{1 / 2}$ & + & - & - & This work \\
${ }^{133} \mathrm{Cs}$ & $23 D_{1 / 2}$ & - & + & + & This work \\
${ }^{133} \mathrm{Cs}$ & $18-37 P_{3 / 2}$ & $+{ }^{\mathrm{b}}$ & - & - & {$[18,21]$} \\
\hline \hline
\end{tabular}

${ }^{\mathrm{a} N o t}$ shown.

${ }^{\mathrm{b}}$ Essentially repulsive $[15,36]$.

state density and a Rydberg fraction of around $2 \%$, this ion density matches $27 \%$ of the Rydberg density.

We then approximate the ionization process with a rate equation. Rydberg atoms with a number density $\mathcal{N}_{\text {Ryd }}$ (assumed constant) collide with ground state atoms with a relative velocity $v$ and a cross-section $\sigma$, contributing to the increase of the density of ions $\mathcal{N}_{\text {ions. }}$. At the same time, ions leave the excitation volume with a rate of $\gamma=1.5 \mathrm{MHz}$, chosen to be the inverse of the transit time through the excitation volume [35]. The corresponding rate equation is

$$
\dot{\mathcal{N}}_{\text {ions }}=\mathcal{N}_{\text {Ryd }}\left(\mathcal{N}_{85}+\mathcal{N}_{87}\right) \sigma v-\gamma \mathcal{N}_{\text {ions }}
$$

and reaches its steady state at

$$
\mathcal{N}_{\text {ions }}=\mathcal{N}_{\text {Ryd }} \frac{\left(\mathcal{N}_{85}+\mathcal{N}_{87}\right) \sigma v}{\gamma} .
$$

Substituting the ion density with the value from the first estimate leads to an ionization cross section of up to $\sigma=$ $1 \times 10^{-3} \mu \mathrm{m}^{2}=0.03 \sigma_{\text {geo }}$, where the geometric cross section of the Rydberg atom is given by $\sigma_{\text {geo }}=\pi\left[\left(n^{*}\right)^{2} a_{0}\right]^{2}$, with an effective quantum number $n^{*}=n-\delta$ and a quantum defect $\delta$. Similar measurements in a pulsed experiment with an atomic beam showed $\sigma=0.06 \sigma_{\text {geo }}$ [29], in very good agreement with our results.

\section{B. Cesium}

Further evidence that the optical bistability is caused by electric fields is found by analyzing the position of the bistability window relative to the unperturbed resonance, which gives the sign of the underlying interaction mechanism. Figures 7(a) and 7(b) show our hysteresis spectra with optical bistability for the $23 \mathrm{D}$ and $28 \mathrm{~S}$ states in ${ }^{133} \mathrm{Cs}$. For both states, the character of the Rydberg-Rydberg interactions is essentially repulsive $[15,36]$. However, the polarizability clearly changes its sign from $\alpha_{23 D}=-0.52 \mathrm{MHz} /(\mathrm{V} / \mathrm{cm})^{2}$ to $\alpha_{28 S}=0.76 \mathrm{MHz} /(\mathrm{V} / \mathrm{cm})^{2}$, as does the position of the bistable region. We summarize these results as well as those from previous measurements in Table I. When comparing the respective observation to the polarizability of each species and angular momentum states, we find perfect agreement with our hypothesis.

Finally, we investigate how the optical bistability is affected by external electric fields. Figures $7(\mathrm{c})-7(\mathrm{~h})$ shows a significant increase in the width of the hysteresis as the frequency of the electric field is varied. The applied electric field amplitudes are small enough that the 

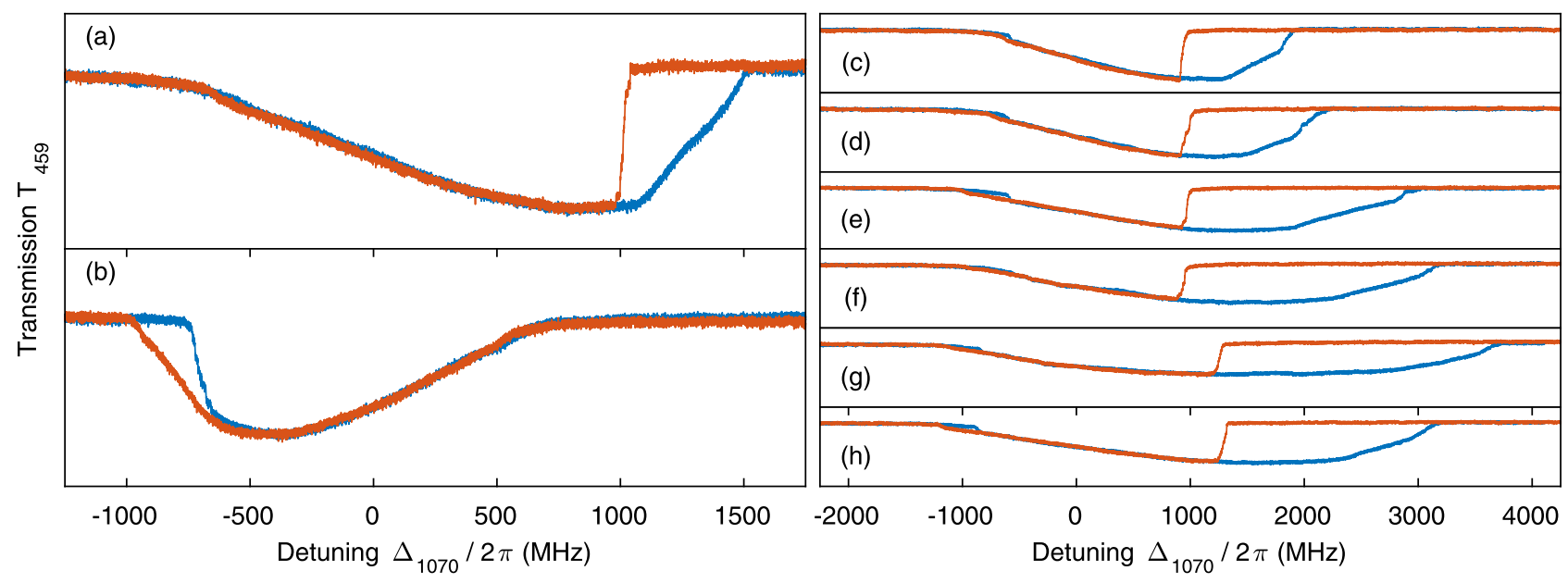

FIG. 7. Cesium measurements. Transmission spectra with frequency scans towards the color of the respective line. (a), (b) Comparison between (a) the $23 \mathrm{D}$ state and (b) the $28 \mathrm{~S}$ state. (c)-(h) Effect on the bistability for the $23 \mathrm{D}$ state by RF modulation with a sine wave of $3.2 \mathrm{~V} \mathrm{~cm}^{-1}$ amplitude and increasing frequency (10, 20, 50, 100, 200, and $500 \mathrm{MHz}$ ).

resulting Stark-shift and the modulation frequency are negligible with regard to the relevant energy scales of the Rydberg atoms. The interparticle interactions of neutral participants are therefore not affected. However, charged particles are heavily influenced. In the range of frequencies used for the electric field modulation (10 to $500 \mathrm{MHz}$ ), the free electrons from the ionization process are accelerated and perform an oscillating trajectory, thus increasing the ionization rate by additional collisions. We believe that this explains why the width of the bistability region increases. Overall, the susceptibility to electric field modulation contradicts a dipoledipole interaction between Rydberg atoms and once more designates that ionization significantly contributes to the observed bistability.

Associated with a significant increase in the width of the hysteresis, a small second hysteresis seems to appear on the opposite detuning sign, observed only with an applied $E$ field and above a certain threshold of frequency and amplitude combination. We are not able to explain this observation at this point or to give more details about the underlying dynamics, and further theoretical and experimental studies are needed in order to fully grasp the microscopics of this complex system. It is worth noticing that a double hysteresis has recently been observed in a related experiment [22].

\section{CONCLUSION}

With the joint examination of two independent atomic species and combinations of principal and azimuthal quantum numbers we are able to preclude Rydberg-Rydberg interactions as the mechanism responsible for the interaction shift in the phenomenon of optical bistability in thermal vapors. At the same time, we find strong evidence that electric fields produced by charges originating from Rydberg ionization are the dominant contribution to the observed effect. Our argumentation based on the results of two different experimental setups contradicts the previously suggested explanations published in Refs. [18,21,22], where the spectrum of experimental parameters overlaps with our settings.
In our first experiment, we have applied two independent EIT schemes, each addressing only one of the naturally abundant isotopes in a rubidium vapor cell. The first scheme strongly drives the transition in ${ }^{85} \mathrm{Rb}$, enabling the atoms to enter a bistable regime, while at the same time, the effect on the ${ }^{87} \mathrm{Rb}$ atoms is monitored by the second EIT scheme. The choice of Rydberg states allows us to exclude dipole-dipole interactions. We find both a deformation of the EIT $_{\mathrm{OB}}$ line and a shift of the EIT $_{\mathrm{FP}}$ line to the red. These observations are in good agreement with the Stark effect caused by electric fields. The fields originate from ionizing collisions of the Rydberg atoms, and the estimated ion densities and the required ionization cross sections are within a realistic range.

In the second experiment using ${ }^{133}$ Cs vapor, we have shown that applying weak electric fields significantly alters the width and position of the bistability. Only charged particles being affected by such weak fields and the distinct accordance of the sign of the polarizability for different states finally support our conclusion.

Further theoretical and experimental investigations are necessary in order to refine the microscopic model for the bistability observed in thermal vapor. For example in Ref. [18], a change in the fluorescence spectrum has been found, which cannot be explained by Stark-shifted Rydberg states or shifts due to (resonant) dipole-dipole interactions alone. This observation is attributed to superradiance by the authors. The connection to bistability however needs further investigation and additional processes have to be included in the model. A more comprehensive understanding of the effects that shift the transition between the bistable states will be beneficial for the progress towards future applications, for example, ac electric field sensing [22,37].

\section{ACKNOWLEDGMENTS}

The research leading to these results has received funding from the Carl-Zeiss Foundation, the European Union's Seventh Framework Programme H2020-FETPROACT-2014 under Grant No. 640378 (RYSQ), and BMBF within Q.Com- 
Q (Project No. 16KIS0129). H.K. acknowledges personal support from the Carl-Zeiss foundation. We appreciate fruitful discussions with T. Pfau and J. P. Shaffer. Thanks to S. Weber, C. Tresp, and S. Hofferberth for providing the code for the computations of pair states and polarizabilities and to $\mathrm{M}$. Abdo and the Institute of Electrical and Optical Communications Engineering for support in developing the microwave circuitry.
[1] M. Saffman and T. G. Walker, Phys. Rev. A 72, 022347 (2005).

[2] M. P. A. Jones, J. Beugnon, A. Gaëtan, J. Zhang, G. Messin, A. Browaeys, and P. Grangier, Phys. Rev. A 75, 040301 (2007).

[3] R. Löw, H. Weimer, U. Krohn, R. Heidemann, V. Bendkowsky, B. Butscher, H. P. Büchler, and T. Pfau, Phys. Rev. A 80, 033422 (2009).

[4] P. Schauß, J. Zeiher, T. Fukuhara, S. Hild, M. Cheneau, T. Macrì, T. Pohl, I. Bloch, and C. Gross, Science 347, 1455 (2015).

[5] D. Maxwell, D. J. Szwer, D. Paredes-Barato, H. Busche, J. D. Pritchard, A. Gauguet, K. J. Weatherill, M. P. A. Jones, and C. S. Adams, Phys. Rev. Lett. 110, 103001 (2013).

[6] Y. Dudin and A. Kuzmich, Science 336, 887 (2012).

[7] T. Peyronel, O. Firstenberg, Q.-Y. Liang, S. Hofferberth, A. V. Gorshkov, T. Pohl, M. D. Lukin, and V. Vuletić, Nature (London) 488, 57 (2012).

[8] D. Tiarks, S. Baur, K. Schneider, S. Dürr, and G. Rempe, Phys. Rev. Lett. 113, 053602 (2014).

[9] H. Gorniaczyk, C. Tresp, J. Schmidt, H. Fedder, and S. Hofferberth, Phys. Rev. Lett. 113, 053601 (2014).

[10] R. Faoro, B. Pelle, A. Zuliani, P. Cheinet, E. Arimondo, and P. Pillet, Nat. Commun. 6, 8173 (2015).

[11] G. Günter, H. Schempp, M. Robert-de Saint-Vincent, V. Gavryusev, S. Helmrich, C. Hofmann, S. Whitlock, and M. Weidemüller, Science 342, 954 (2013).

[12] D. Barredo, H. Labuhn, S. Ravets, T. Lahaye, A. Browaeys, and C. S. Adams, Phys. Rev. Lett. 114, 113002 (2015).

[13] H. Schempp, G. Günter, M. Robert-de Saint-Vincent, C. S. Hofmann, D. Breyel, A. Komnik, D. W. Schönleber, M. Gärttner, J. Evers, S. Whitlock, and M. Weidemüller, Phys. Rev. Lett. 112, 013002 (2014).

[14] N. Malossi, M. M. Valado, S. Scotto, P. Huillery, P. Pillet, D. Ciampini, E. Arimondo, and O. Morsch, Phys. Rev. Lett. 113, 023006 (2014).

[15] A. Urvoy, F. Ripka, I. Lesanovsky, D. Booth, J. P. Shaffer, T. Pfau, and R. Löw, Phys. Rev. Lett. 114, 203002 (2015).

[16] V. Bendkowsky, B. Butscher, J. Nipper, J. P. Shaffer, R. Löw, and T. Pfau, Nature (London) 458, 1005 (2009).

[17] J. A. Sedlacek, A. Schwettmann, H. Kübler, and J. P. Shaffer, Phys. Rev. Lett. 111, 063001 (2013).
[18] C. Carr, R. Ritter, C. G. Wade, C. S. Adams, and K. J. Weatherill, Phys. Rev. Lett. 111, 113901 (2013).

[19] M. Marcuzzi, E. Levi, S. Diehl, J. P. Garrahan, and I. Lesanovsky, Phys. Rev. Lett. 113, 210401 (2014).

[20] N. Šibalić, C. G. Wade, C. S. Adams, K. J. Weatherill, and T. Pohl, Phys. Rev. A 94, 011401 (2016).

[21] N. R. de Melo, C. G. Wade, N. Šibalić, J. M. Kondo, C. S. Adams, and K. J. Weatherill, Phys. Rev. A 93, 063863 (2016).

[22] D. Ding, C. Adams, B. Shi, and G. Guo, arXiv:1606.08791.

[23] A. K. Mohapatra, T. R. Jackson, and C. S. Adams, Phys. Rev. Lett. 98, 113003 (2007).

[24] S. Shepherd, D. J. Fulton, and M. H. Dunn, Phys. Rev. A 54, 5394 (1996).

[25] A. Urvoy, C. Carr, R. Ritter, C. Adams, K. Weatherill, and R. Löw, J. Phys. B 46, 245001 (2013).

[26] P. Thoumany, T. Hänsch, G. Stania, L. Urbonas, and T. Becker, Opt. Lett. 34, 1621 (2009).

[27] A. Schwettmann, J. Crawford, K. R. Overstreet, and J. P. Shaffer, Phys. Rev. A 74, 020701 (2006).

[28] E. A. Goldschmidt, T. Boulier, R. C. Brown, S. B. Koller, J. T. Young, A. V. Gorshkov, S. L. Rolston, and J. V. Porto, Phys. Rev. Lett. 116, 113001 (2016).

[29] G. Vitrant, J. Raimond, M. Gross, and S. Haroche, J. Phys. B 15, L49 (1982).

[30] D. Barredo, H. Kübler, R. Daschner, R. Löw, and T. Pfau, Phys. Rev. Lett. 110, 123002 (2013).

[31] T. F. Gallagher, Rydberg Atoms, Cambridge Monographs on Atomic, Molecular and Chemical Physics, Vol. 51 (Cambridge University, Cambridge, England, 1999), pp. 143-188.

[32] J. Holtsmark, Ann. Phys. 363, 577 (1919).

[33] M. Fleischhauer, A. Imamoglu, and J. P. Marangos, Rev. Mod. Phys. 77, 633 (2005).

[34] D. Hummer, J. Quant. Spectrosc. Radiat. Transfer 36, 1 (1986).

[35] W. Demtröder, in Laserspektroskopie 1: Grundlagen (Springer, Berlin, 2011), pp. 43-68.

[36] K. Singer, J. Stanojevic, M. Weidemüller, and R. Côté, J. Phys. B 38, S295 (2005).

[37] H. Fan, S. Kumar, J. Sedlacek, H. Kübler, S. Karimkashi, and J. P. Shaffer, J. Phys. B 48, 202001 (2015). 